\section{Aseptic Gingivitis Related to Quetiapine Hemifumarate}

\section{Gahr, M. A. Kölle, R. W. Freudenmann, C. Schönfeldt-Lecuona}

Department of Psychiatry and Psychotherapy III, University

Hospital of Ulm, Ulm, Germany

\section{Abstract \\ $\nabla$}

Quetiapine hemifumarate (QF) is widely used in psychiatry and is associated with regularly occurring side effects such as dizziness and metabolic problems. Apart from these typical adverse events the agent has attracted attention for several rare phenomena (priapism, cholestasis, rhabdomyolysis) that indeed feature anecdotal character, but are nevertheless indispensable for a comprehensive understanding of the factual risk profile of quetiapine. We present the first report of aseptic gingivitis associated with $\mathrm{QF}$ in a patient with mental retardation.

\section{Key words}

adverse events · antipsychotic treatment. inflammation · second generation antipsychotics side effects

\section{Introduction}

Quetiapine hemifumarate (QF), that is available as a nonretarded as well as a retarded preparation, is a common agent in psychiatry with a wide spectrum of indications ranging from schizophrenia to bipolar disorder [1]. It has been assessed to be effective for its main indication in schizophrenia and is frequently associated with side effects such as dizziness, dry mouth, weight gain and sleepiness [2]. Apart from these typical adverse events there are anecdotal reports of rare adverse events related to quetiapine such as dependence [3], rhabdomyolysis [4], priapism [5], cholestasis [6], and hypothyroidism [7] that expand the knowledge of treatment-dependent risks. In the following we report the first case of QF-associated aseptic gingivitis.

\section{Case Report}

$\nabla$

The 34-year-old female patient was treated at regular intervals in our outpatient department for agitation and severe psychomotor arousals related to moderate mental retardation accompanied by generalized idiopathic epilepsy. Following impaired psychomotor and speech development and a first generalized tonic clonic seizure at the age of 3 years the patient was hospitalized in several neurological and later on psychiatric departments for diagnostic reasons. Considering normal pregnancy and delivery as well as unremarkable findings regarding several magnetic resonance imagings of the brain, the patient's phenotype, examination of cerebrospinal fluid and chromosome analysis, the etiology of the patient's syndrome was finally classified as unidentified. Further anamnesis revealed absence of other psychiatric or neurological diseases and there was no history of alcohol, nicotine or illicit drug use. The patient's family history was unremarkable. Subsequent to frequently occurring severe agitation and aggressiveness due to the patient's high irritability, a psychiatric treatment was established in order to particularly ameliorate these events. At the time of the first treatment in our outpatient department, 4 years prior to the reported episode, the patient was treated with valproic acid $(1000 \mathrm{mg}$ per day), oxcarbazepine (1500 mg per day), haloperidol (5 mg per day), mirtazapine ( $15 \mathrm{mg}$ per day), prothipendyl ( $40 \mathrm{mg}$ per day) and biperiden ( $4 \mathrm{mg}$ per day). Anticonvulsive therapy with valproic acid and oxcarbazepine was present for approximately 7 years resulting in complete suppression of epileptic seizures and without the occurrence of adverse events. In the further course we modified the medication and could achieve a substantial improvement regarding agitation and paroxysmal arousals with QF (400 mg per day, retarded preparation), haloperidol (3 mg per day) and valproic acid ( $1000 \mathrm{mg}$ per day). Termination of oxcarbazepine treatment did neither result in worsening of electroencephalographic findings nor in an occurrence of seizures. After approximately 1 year of stable remission regarding psychomotor target symptoms, the patient's legal guardian reported restlessness and increase of agitation. Thus, QF (retarded preparation) was slowly titrated up to $800 \mathrm{mg}$ per day within 2 weeks until the patient presented a sufficient improvement. After approximately 1 week of treatment with elevated QF ( $800 \mathrm{mg}$ per day) the patient developed painful gingivitis discontinuously affecting the gingiva of the maxilla and mandible, which was primarily not connected with the dose escalation. There was no previous hypertrophy of the gum tissue. Considering the unremarkable blood examination (including inflammatory parameters, differential blood count, liver enzymes, serum vitamin C levels, no pregnancy by means of blood human chorionic gonadotropin levels) and dental examination, hygiene measures (antimicrobial mouth rinses and thorough dental care after food intake) were performed for another 2 weeks without amelioration of the gingival inflammation. There was no temporal link between the onset of gingivitis and the patient's menstrual cycle and the patient did not take any oral contraception. Clinical signs indicating an oral fungal infection were absent. Since gingivitis developed subsequent to increased QF dose and all other relevant parameters (unchanged dosages of haloperidol and valproic acid, unaltered nutritional habits) remained unchanged QF was reduced probatorily down to $400 \mathrm{mg}$ per day. Within 1 week the gingival inflammation subsided completely but the patient relapsed regarding psychomotor agitation as expected and lorazepam was administered temporarily. As a second probatory intervention $\mathrm{QF}$ was increased again up to $800 \mathrm{mg}$ per day and gingival inflammation reoccurred. Hence we kept QF (retarded preparation) at a dosage of $400 \mathrm{mg}$ per day and established an additional medication with promethazine ( $50 \mathrm{mg}$ thrice per day) with which we achieved an improvement of agitation. Again gingival inflammation ceased within 1 week and has not relapsed since the last 6 months.

\section{Discussion \\ $\nabla$}

In the presented case the gingival inflammation occurred with a close temporal relation to elevated QF doses. This phenomenon subsided after dose reduction and reoccurred after rechallenge with the increased dose. Application of the Naranjo adverse drug reaction probability scale [8] to this constellation reveals a score of at least 5 , indicating a probable causation of the observed 
gingivitis by QF. Since other conclusive factors that could explain our observation are lacking, we think that QF might have contributed to the development of gingivitis in a causal way. Gingivitis and gingival enlargement are known as side effects of a treatment with antiepileptic drugs (especially carbamazepine, valproic acid and phenytoin) [9]. However, the pathomechanisms underlying these phenomenona are not completely elucidated [10]. Taking into account that gingivitis did not occur under a 7-year treatment with oxcarbamazepine and valproic acid, it is not plausible to hypothesize that gingivitis is a consequence of continued treatment with valproic acid, particulary in consideration of the observed dose dependence. Considering that other reports regarding similar observations are lacking, the etiology of this phenomenon remains fairly speculative. In the present case, the absence of a detectable systemic inflammation and unremarkable differential blood count suggest an aseptic reaction that seems to be dose-dependent and potentially idiopathic since gingivitis did not occur under lower doses of QF.

\section{Conflict of Interest}

$\nabla$

The authors declare no conflicts of interest.

\section{References}

1 Wang Z, Kemp D, Chan $P$ et al. Comparison of the tolerability and sensitivity of quetiapine-XR in the acute treatment of schizophrenia, bipolar mania, bipolar depression, major depressive disorder, and generalized anxiety disorder. Int J Neuropsychopharmacol 2011; 14 : 131-142

2 Srisurapanont M, Maneeton B, Maneeton N. Quetiapine for schizophrenia. Cochrane Database Syst Rev 2004: CD000967

3 Yargic I, Caferov C. Quetiapine dependence and withdrawal: a case report. Subst Abus 2011; 32: 168-169

4 Ceri M, Unverdi S, Altay M et al. Comment on: low-dose quetiapineinduced severe rhabdomyolysis. Ren Fail 2011; 33: 463-464

5 Tsai A. A case of recurrent priapism in the context of quetiapine use, discontinuation, and rechallenge. J Clin Psychopharmacol 2011; 31: 235-236

6 Klinke J, Shapira S, Akbari E et al. Quetiapine-associated cholestasis causing lipoprotein-X and pseudohyponatraemia. J Clin Pathol 2010; 63: 741-743

7 Poutanen O, Iso-Koivisto E, Työläjärvi $M$ et al. Quetiapine-associated hypothyroidism in young female patients: a report of three cases. Pharmacopsychiatry 2010; 43: 237-239

8 Naranjo C, Busto $U$, Sellers $E$ et al. A method for estimating the probability of adverse drug reactions. Clin Pharmacol Ther 1981; 30 : 239-245

9 Galas-Zgorzalewicz B, Borysewicz-Lewicka M, Zgorzalewicz $M$ et al. The effect of chronic carbamazepine, valproic acid and phenytoin medication on the periodontal condition of epileptic children and adolescents. Funct Neurol 1996; 11: 187-193

10 Seymour R, Thomason J, Ellis J. The pathogenesis of drug-induced gingival overgrowth. J Clin Periodontol 1996; 23: 165-175

$\begin{array}{ll}\text { received } & 18.05 .2012 \\ \text { revised } & 08.07 .2012 \\ \text { accepted } & 11.07 .2012\end{array}$

\section{Bibliography}

DOI http://dx.doi.org/

10.1055/s-0032-1321906

Published online ahead of print:

22 August 2012

Pharmacopsychiatry 2013;

46: $39-40$

(c) Georg Thieme Verlag KG

Stuttgart · New York

ISSN 0176-3679

\section{Correspondence}

\section{Dr. M. Gahr}

Department of Psychiatry and Psychotherapy III University Hospital of Ulm

Leimgrubenweg 12-14

89075 Ulm

Germany

Tel.: +49/731/50061552

Fax: $+49 / 731 / 50061452$

maximilian.gahr@uni-ulm.de 